\title{
Wax pile method for glioma surgery utilizing intraoperative magnetic resonance imaging: a technical note
}

\author{
Toshihide Tanaka ${ }^{1 *}$, Takuya Ishii ${ }^{4}$, Naoki Kato ${ }^{1}$, Takao Arai ${ }^{2}$, Yasuharu Akasaki ${ }^{2}$, Yohei Yamamoto ${ }^{3}$, Yuzuru Hasegawa ${ }^{1}$ and Toshiaki Abe ${ }^{3}$ \\ *Correspondence: ttanaka@jikei.ac.jp \\ 'Department of Neurosurgery, Jikei University School of Medicine, Kashiwa Hospital, Kashiwa, Chiba, Japan. \\ ${ }^{2}$ Department of Neurosurgery, Jikei University School of Medicine, Katsushika Medical Center, Tokyo, Japan. \\ ${ }^{3}$ Department of Neurosurgery, Jikei University School of Medicine, Tokyo, Japan. \\ ${ }^{4}$ Department of Neurosurgery, Atsugi City Hospital, Atsugi, Kanagawa, Japan.
}

\begin{abstract}
Over the past decade, intraoperative magnetic resonance imaging (iMRI) to facilitate precise resection of brain tumors has been introduced to the field of brain tumor surgery. We have started using a technique for brain tumor resection involving precise placement of silicone tubes filled with bone wax under iMRI, which we call the "wax pile" method. From July 2008 to August 2012, a total of 31 patients with glioma were surgically treated by craniotomy utilizing iMRI. The wax pile method was used to treat 16 patients with glioma, including 9 cases of glioblastoma, 2 cases of anaplastic astrocytoma, 4 cases of oligodendroglioma, and 1 case of low-grade astrocytoma. To determine the resection margins, 4-6 tubes were inserted around the tumor and tube positions were confirmed on iMRI. Extent of tumor removal was total in 10 cases $(62.5 \%)$ and partial $(<95 \%$ removal) in 6 cases (37.5\%). "Wax piles" with clear contours and sharp neuroimages were quite useful as intraoperative markers for determining the depth and range of the lesions under iMRI. Tumors were removed safely and accurately. Karnofsky performance status (KPS) score ranged from 40 to 100 preoperatively, and was not deteriorated postoperatively in any case. No complications due to tube insertion were encountered. The present study demonstrated that the wax pile method offers a safe, accurate technique for glioma surgery without concern for brain shift during tumor resection under iMRI.
\end{abstract}

Keywords: Glioma, intraoperative magnetic resonance imaging, intraoperative marker, surgery, wax pile method, wax plate

\section{Introduction}

To perform precise resection and avoid functional deterioration after surgery, assistance devices such as neuronavigation systems, neurophysiological monitoring, chemical fluorescence and intraoperative magnetic resonance imaging (iMRI) have been developed [1-5]. The use of iMRI has gradually gained popularity since the first operation utilizing a 0.5-T open MRI was introduced at Brigham and Women's Hospital $[4,6]$.

Neither navigation systems nor even iMRI provide realtime imaging of the range of resection, particularly in terms of the depth of surgical access to the tumor during resection. A navigation-guided fence-post tube procedure has been introduced [7,8], in which the target area of resection is defined by fence posts inserted under guidance of the navigation system. However, a key problem with this approach is that brain shift occurs during tumor resection. If additional resection is required to remove the residual tumor, a navigation-guided procedure alone is inadequate.

As a more convenient and advanced technique to provide true real-time navigation useful for precisely assessing tumor margins and residual tumor, we tried to establish intraoperative markers under iMRI utilizing silicone tubes filled with bone wax, which we termed a "wax pile" and the flattened bone wax a "wax plate".

The present study introduces this technique utilizing iMRI to perform precise resection of brain tumors, particularly lowgrade astrocytomas. Wax piles and wax plates inserted around the tumor after dural incision allowed ready visualization of the resection plane under iMRI. The materials used are safe and inexpensive. The present study evaluated our experience with the use of wax piles and wax plates to obtain precise delineation of the resection margin of the tumor and examined the accuracy of using these intraoperative markers according to neuroradiological and histological findings.

\section{Patients and methods \\ Patient population}

Between July 2008 and August 2012, a total of 31 patients with glioma were surgically treated by craniotomy utilizing iMRI. Of these, 16 patients underwent surgery with the wax pile method. The tumor was graded in accordance with World Health Organization classification as low-grade astrocytoma (LGA) in 1 case, oligodendroglioma (OLIG) in 4 cases, anaplastic astrocytoma (AA) in 2 cases, and glioblastoma multiforme (GBM) in 9 cases. Characteristics of all patients are shown in Table 1. Karnofsky performance scale (KPS) score was compared between pre- and postoperative status [9].

The ethics committee at Jikei University School of Medicine approved the protocol for craniotomy with iMRI, and informed consent was obtained from each patient prior to surgery with iMRI. 
Tanaka et al. Neuroscience Discovery 2013,

http://www.hoajonline.com/journals/pdf/2052-6946-1-6.pdf

doi: $10.7243 / 2052-6946-1-6$

Table 1. Summary of the patients underwent craniotomy using intraoperative MR images with "wax piles".

\begin{tabular}{llllllllll}
\hline Case No. & Age & Sex & Diagnosis & Location & Removal & No. of piles & Additional pile/plate & Preope. KPS & Postope. KPS \\
\hline 1 & 45 & M & AA & Lt. frontal & total & 4 & none & 100 & 100 \\
2 & 59 & F & GBM & Rt. frontal & partial & 4 & none & 40 & 40 \\
3 & 59 & M & GBM & Rt. temporal & total & 4 & 1 plate & 100 & 100 \\
4 & 50 & F & OLIG & Lt. frontal & partial & 4 & none & 100 & 100 \\
5 & 78 & M & GBM & Rt. temporal & total & 4 & 2 piles/1 plate & 50 & 50 \\
6 & 36 & M & OLIG & Lt. parietal & partial & 1 & none & 100 & 100 \\
7 & 41 & F & OLIG & Rt. frontal & total & 6 & 2 piles/1 plate & 100 & 100 \\
8 & 25 & F & AA & Rt. parietal & partial & 1 & none & 100 & 100 \\
9 & 64 & M & GBM & Rt. parietal & total & 6 & 4 piles/1 plate & 100 & 100 \\
10 & 49 & M & GBM & Lt. frontal & total & 4 & 1 plate & 100 & 100 \\
11 & 45 & F & LGA & Lt. frontal & total & 4 & none & 100 & 100 \\
12 & 58 & M & OLIG & Rt. frontal & total & 4 & none & 100 & 100 \\
13 & 63 & M & GBM & Lt. frontal & subtotal & 2 & none & 90 & 90 \\
14 & 67 & F & GBM & Rt. parietal & subtotal & 2 & none & 60 & 60 \\
15 & 77 & F & GBM & Lt. temporal & total & 3 & none & 70 & 80 \\
16 & 66 & M & GBM & Rt. temporal & total & 3 & none & 100 & 100 \\
\hline
\end{tabular}

$\mathrm{M}=$ male; $\mathrm{F}$ = female; KPS = Karnofsky Performance Scale; LGA = low grade astrocytoma;

$\mathrm{GBM}=$ glioblastoma multiforme; OLIG = oligodendroglioma; $\mathrm{OA}=$ oligoastrocytoma;

$\mathrm{AA}=$ anaplastic astrocytoma; $\mathrm{AOA}=$ anaplastic oligoastrocytoma; $\mathrm{Lt}=\mathrm{left} ; \mathrm{Rt}=\mathrm{right}$

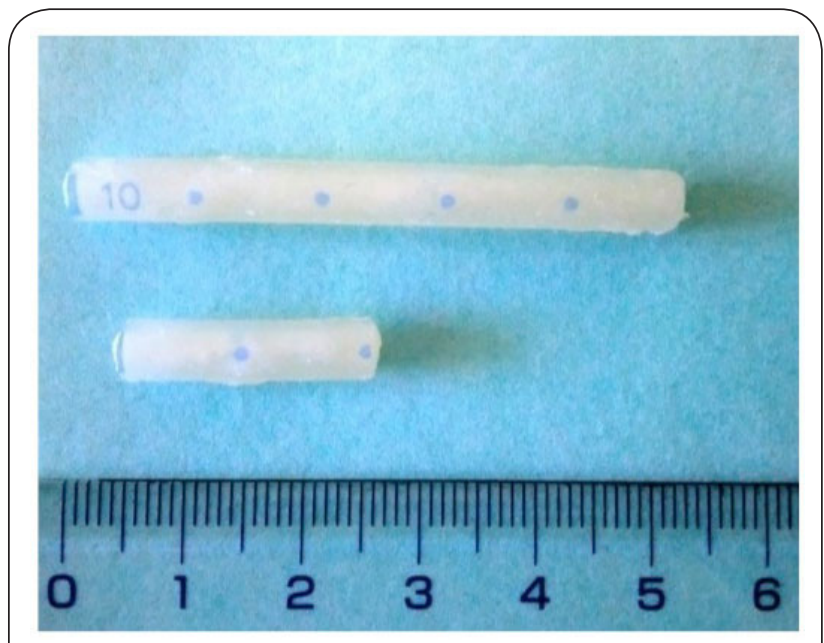

Figure 1. Photograph demonstrating silicone catheters filled with bone wax used as "wax piles". Before tumor resection, long piles were used. Silicone tubes were filled with the melted bone wax placed into a sterilized tube in boiled water by aspiration. Silicone tubes containing bone wax were then cut in pieces.

\section{Intraoperative procedure}

The iMRI was performed using a 0.7-T Altaire (Hitachi Medico, Kashiwa, Japan) MRI operation system situated adjacent to the operating room ${ }^{2}$. The head of the patient was fixed in a Sugita frame after induction of general anesthesia. In the beginning, silicone tubes $(5 \mathrm{~mm}$ in diameter, E-8L containing bone wax (at approximately $95^{\circ} \mathrm{C}$ ) were prepared. In brief, a small piece of the bone wax was placed in the sterilized tube and soaked in boiling water to let the bone wax liquefy. Subsequently, a silicone tube was inserted into the sterilized tube containing the bone wax and filled with the melted bone wax by aspiration. In the end, the silicone tube containing bone wax was cut in pieces, forming the wax piles (Figure 1). The lengths of the tubes were dependent on the depth of resection required for tumor removal. Craniotomy and dural incision were performed in the usual fashion (Figure 2A). After dural incision, four wax piles were placed around the tumor and inserted toward the bottom of the tumor under ultrasonographic guidance to clearly visualize the tumor margins (Figures $2 \mathrm{C}$ and $\mathbf{2 E}$ ). The number and location of tubes was dependent on the distribution of the cortical veins, fissures, sulci and gyri. Between 4 and 6 wax piles were inserted, depending on the size of the tumor (Table 1). If the tumor included an eloquent area or was located close to an eloquent region, wax piles were placed within the tumor. We had to refrain from inserting tubes into eloquent regions to avoid postoperative neurological deficit due to injury from tube insertion. In cases of tumors close to the pyramidal tract, we routinely performed SEP/MEP (sensory evoked potential/motor evoked potential) monitoring, then iMRI was examined to determine whether tube placement corresponded to tumor margins (Figure 2D).

Wax piles showed dark signal intensity on T2-weighted or fluid-attenuated inversion recovery (FLAIR) imaging when compared with tumor or normal brain tissue (Figure 2D). When the surgeon thought that gross total removal of the tumor had been achieved, or considered that more information was needed concerning the extent of resection and residual tumor as detected by 5-aminolevulinic acid (5-ALA), a wax plate (i.e., a piece of flattened bone wax) was placed on 


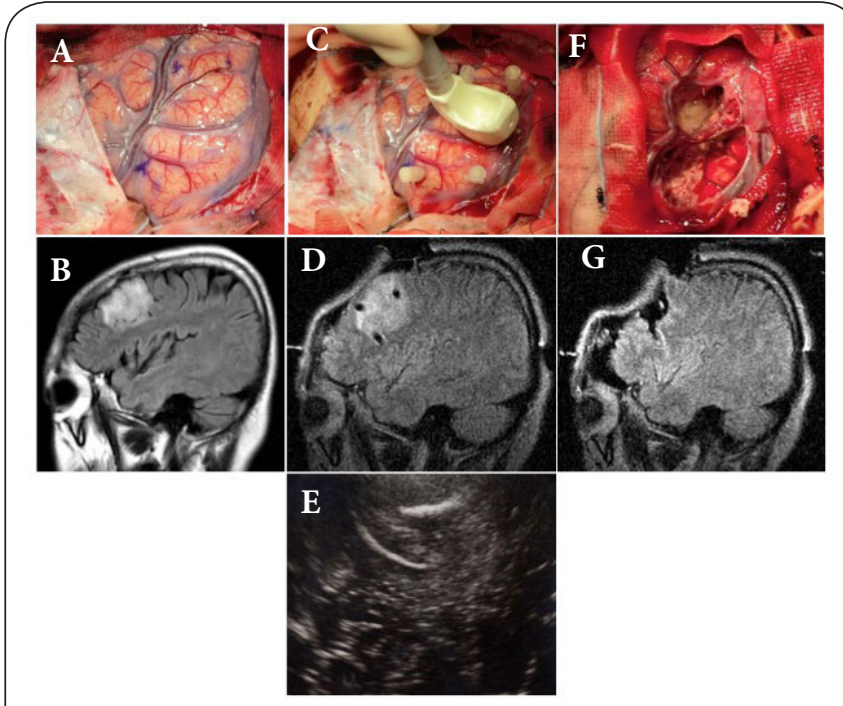

Figure 2. Representative intraoperative photographs (A, C, E) and fluid-attenuated inversion recovery (FLAIR) images (B, D, G) in Case 12. Preoperative MRI shows a high-intensity mass in the right frontal lobe (B). After dural incision, the margins of the tumor are difficult to detect (A), then four wax piles are inserted at the tumor margin $(\mathbf{C})$ with the assistance of ultrasonography (E). After insertion of wax piles, FLAIR images clearly demonstrate the anterior and posterior piles inserted at the tumor margins (D). Intraoperative photograph shows resected cavity after tumor removal (F). Complete removal of the tumor is confirmed before dural closure (G).

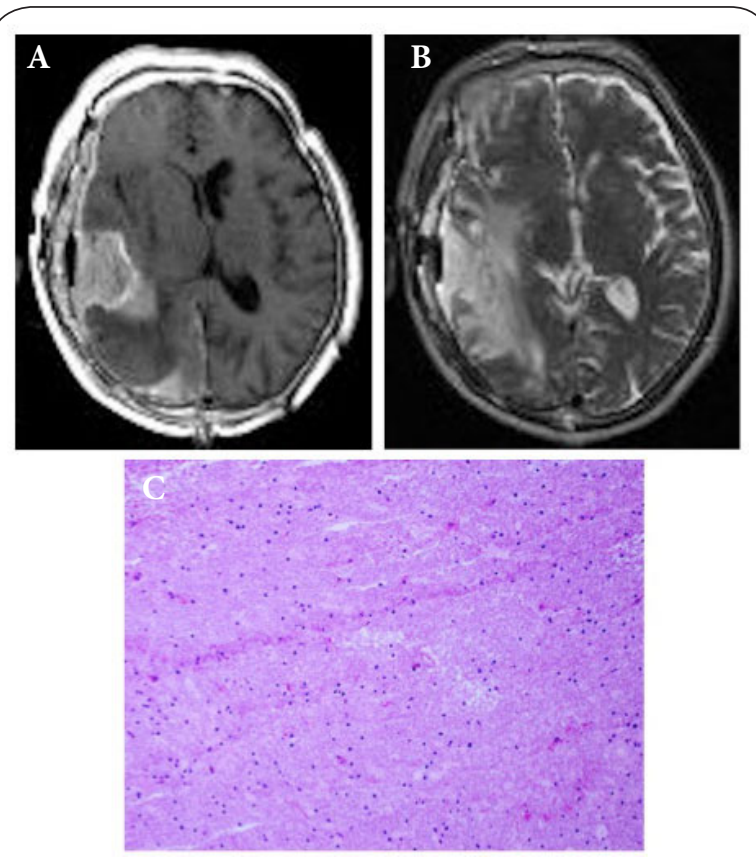

Figure 4. Case 5. Before dural closure, complete removal of the tumor is confirmed by final intraoperative T1-weighted imaging without gadolinium enhancement (A) and T2-weighted MRI (B). Residual tumor was not identified in the resected cavity by pathological findings (C).

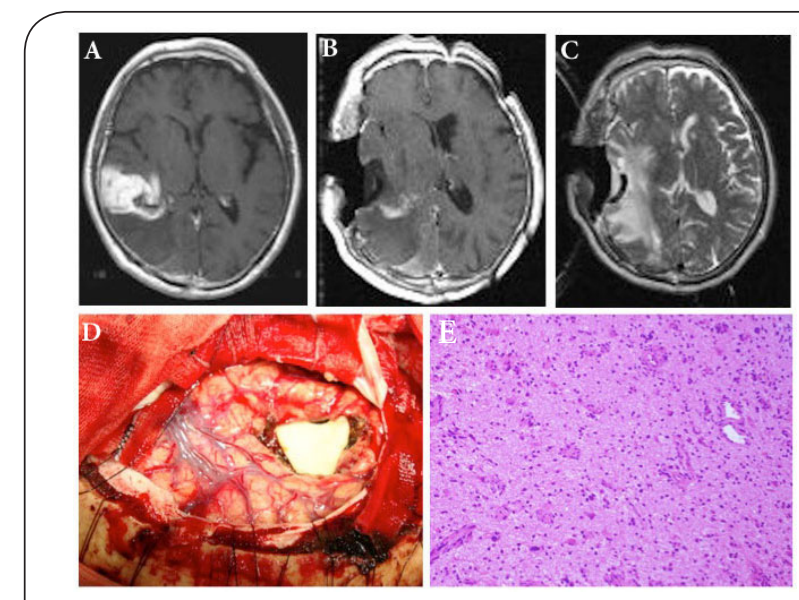

Figure 3. Representative MRI, intraoperative photographs and microphotographs from Case 5. Preoperative T1weighted MRI with gadolinium show a strongly enhanced mass in the right temporal lobe (A). After tumor resection, a wax plate is placed at the location corresponding to a positive area of fluorescent reaction by 5 -aminolevulinic acid (5-ALA) (D). T1-weighted gadolinium-enhanced and T2-weighted MRI demonstrated the wax plate as an area of dark intensity in the resected cavity $(\mathbf{B}, \mathbf{C})$. Residual tumor is identified beneath the "wax plate" (low-intensity band) (B). Micrograph demonstrates a high density of tumor cells and microvessels in the section obtained from tissue beneath the wax plate $(\mathbf{E})$. the resection margin or 5-ALA-positive area (Figure 3D). Then iMRI was then performed using the same sequence as preoperative MRI. Residual tumors in the resection cavity were easily detected using wax plates (Figures $\mathbf{3 B}$ and $\mathbf{3 C}$ ). Complete removal of residual tumors was confirmed by both iMRI (Figures 4A and 4B) and histological findings from rapid frozen sections (Figure 4C).

\section{Results}

In all cases, initial iMRI identified tumor margins using wax piles before resection. The extent of tumor removal was total in 10 cases $(62.5 \%)$ and partial (i.e., $<95 \%$ removal) in 6 cases (37.5\%, Table 1). Among the 6 cases with partial removal, tumors were located adjacent to the motor area in 4 cases. One of the remaining two tumors had invaded into Broca's area, and the other was located in the basal ganglia associated with invasion to the contralateral side via the corpus callosum. Additional surgical removal using wax piles or wax plates following the initial tumor resection and iMRI was required in 5 cases (31.3\%, Table 1$)$.

The period of interruption for each iMRI session was approximately 30-40 min, depending on the imaging seq-uences applied. Additional resection was performed whenever possible. Residual tumors with short wax piles or wax plates were easily visualized (Figure 2D, Figures 3B and 3C). 
Tanaka et al. Neuroscience Discovery 2013,

Histological findings from rapid frozen sections clearly demonstrated the presence of tumor cells from tissue beneath the wax plate (Figure 3D and $\mathbf{3 E}$ ) and the absence of tumor cells from the resection cavity (Figure 4C).

KPS scores ranged from 40 to 100 before surgery, and no postoperative deterioration in KPS score was seen in any case (Table 1). No incidents due to tube insertion were encountered, and no cases of infection or postoperative hemorrhage occurred.

\section{Representative cases}

Case 12: A 58-year-old man with right frontal oligodendroglioma presented with loss of consciousness and was referred to our institute. He underwent right frontal craniotomy in the usual fashion (Figure 2A). FLAIR imaging revealed a high-intensity mass in the right frontal lobe (Figure 2B). After dural incision, four wax piles were inserted under ultrasonographic assistance at the margin where the softness of the brain tissue varied, although tumor margins were obscure (Figures $2 \mathrm{C}$ and $2 \mathrm{E}$ ). The wax pile positions were confirmed by iMRl, clearly demonstrating that the piles had been inserted into the anterior and posterior margins of the tumor and that the depth of wax piles reached exactly to the bottom margin of the tumor (Figure 2D). The tumor could be partially removed with the assistance of wax piles as indicators of tumor margins. After tumor removal (Figure 2F), iMRI revealed the actual extent of tumor removal (Figure 2G).

Case 5: A 78-year-old man with right temporal glioblastoma multiforme was referred to our institute with left hemiparesis and left homonymous hemianopsia. MRI demonstrated an enhanced mass in the right temporal lobe (Figure 3A). Right temporal craniotomy was performed. Tumor margins were quite obvious after dural incision. Wax piles were inserted at the tumor margins. After tumor removal, presence of residual tumor was examined using 5-ALA. A wax plate was placed at the site of the 5-ALA-positive area (Figure 3D). Second iMRI showed a residual enhanced mass beneath the wax plate (Figure 3B). T2-weighted MRI clearly revealed the wax plate as a region of dark intensity (Figure $3 \mathrm{C}$ ). Histological findings from rapid frozen sections of tissue from beneath the wax plate demonstrated a high density of tumor cells and microvessels (Figure 3E). Further resection of the tumor was therefore performed using the updated information. Following additional resection, third iMRI showed complete removal of the tumor (Figures $4 \mathrm{~A}$ and $\mathbf{4 B}$ ). Histological findings identified no residual tumor along the resection plane (Figure 4C).

\section{Discussion}

The combination of iMRI with navigation systems has been used for brain tumor surgery, and many reports have described their efficacy [1-6,10]. However, navigation systems cannot provide real-time information due to brain shifts caused by gravity, brain retraction, aspiration of cerebrospinal fluid, and reduction of the tumor mass. Brain shift is influenced by tissue characteristics, intraoperative positioning of the patient, opening of the ventricular system, craniotomy size, and resection volume. The maximum shift in white matter bundles has been reported to range from -8 to $+15 \mathrm{~mm}$ intraoperatively, and the direction of shift is unpredictable [11]. Another report described the magnitude of pyramidal tract displacement due to removal of tumor as varying from 0.5 to $8.7 \mathrm{~mm}$ on the lesion side and from 0 to $3.6 \mathrm{~mm}$ on the normal side [12]. Use of an ultrasound-linked navigation system or updating navigation with intraoperative images have been used to compensate for brain shift [13]. However, all these procedures are cumbersome. To overcome these problems, intraoperative markers that are not influenced by brain shift are needed.

As a more convenient technique to realize true real-time navigation useful for precisely assessing tumor margins, fluorescent navigation using 5-ALA has recently been proposed [14]. However, this method also has problems. For one thing, the intensity of fluorescence varies among patients, especially with low-grade glioma.

Ahn et al., reported the usefulness of gadolinium-soaked cotton pledgets to determine the resection margins of pituitary tumors using iMRI during transsphenoidal surgery [15]. These contrast-soaked cotton pledgets appeared as areas of dark intensity compared with surrounding structures and allowed clear delineation of the tumor resection margins.

Kajiwara [7] and Yoshikawa et al., [8] described navigationguided fence-post tube technique under the guidance of navigation systems. They inserted fence-post tubes before dural incision to avoid brain shift as much as possible. When an intraoperative navigation system is not used, we believe that opening of the dura is inevitable for inserting intraoperative markers such as fence-post tubes, since confirmation of cortical veins and tumor location is necessary to avoid injury. On the other hand, Ohue et al., reported inserting four silicone catheters at the tumor margin after dural incision. Following insertion of the catheters, ultrasound-linked navigation clearly demonstrated the location of the catheter as a real-time image [13]. However, the problem with this approach is that some tumors show only fair or poor ultrasound images, making residual tumor difficult to evaluate using a synchronized navigation system during surgery.

The present study used the wax pile method to overcome the problem of brain shift and neuroimage quality, as described above. Silicone tubes filled with bone wax, which we termed wax piles, were inserted along the tumor margin, providing real-time intraoperative markers as useful navigation aids during surgery. In addition, the wax pile is hyperechoic and easily visualized on intraoperative ultrasound without distortion and equally well visualized and easily distinguished from tumor on iMRI. Obviously, the quality of images is better from MRI than from ultrasound. In addition, our current technique utilizing wax piles and wax plates under iMRI is safe and accurate. Using wax piles, the position of the resection 
margin and depth of the tumor can be confirmed immediately after the procedure on iMRI. This makes the area to be resected more visible and the margins and depth of resection easier to determine without hesitation.

The properties of wax make it a very good tool for intraoperative use, as opposed to metallic objects such as titanium or other substances. We tried to use a silicone tube filled with contrast medium diluted to various concentrations, but this was not clearly visible and did not prove useful as an intraoperative marker (data not shown). Titanium plates occasionally cause image distortion. In addition, the ethics committee placed restrictions on the intraoperative use of materials. Based on these findings, we believe that bone wax represents the most appropriate material for use as an intraoperative marker of iMRI.

In terms of the advantages of using bone wax as a real-time intraoperative marker, most tumors appeared hyperintense on FLAIR and T2-weighted imaging, so a material showing low intensity was considered useful for accentuating FLAIR or T2 contrast. Ahn et al., also noted that bone wax in transsphenoidal surgery was not always reliable in differentiating resection margins from surrounding structures [15]. However, bone wax used in the method described here was sufficiently thick allowing easy visualization on $\mathrm{iMRI}$ sequences. The wax piles were inserted after dural incision and the location confiemed by iMRI. Evaluating the cortex before inserting the wax pile is important and neuro-navigation, ultrasound, or iMRI used to assess the tumor before inserting the wax piles. This technique was useful to evaluate the location and depth of residual tumor.

Location of the inserted tubes that were more clearly visualized by iMRI provided quite useful information regarding the degree of intraoperative brain shift after dural incision and debulking of the tumor, particularly when additional resection was necessary.

Using the wax pile and wax plate method under iMRI, lesions could be determined precisely and removed completely. Surgeons could decide on further resection of invisible residual tumors without hesitation or confusion. Moreover, no complications associated with the use of wax piles and wax plates were encountered. The wax pile sites were confirmed following SEP/MEP monitoring in the case of tumors located in eloquent areas. To avoid cortical injury, wax piles were first inserted into the tumor tissue.

Silicone tubes and bone wax are frequently used in standard operations. Not only are the safety of these materials well known, but procedures using silicone tube and bone wax also involve minimal cost.

The present results demonstrated the accuracy and safety of the wax pile method for brain tumor surgery even by surgeons without abundant experience, or even when an intraoperative navigation system cannot be used. The wax pile method under iMRI provided real-time information about the direction of resection, as well as the extent of tumor resection during surgery.

\section{Competing interests}

The authors declare that they have no competing interests.

Authors' contributions

\begin{tabular}{|l|c|c|c|c|c|c|c|c|}
\hline Authors' contributions & TT & TI & NK & TA1 & YA & YY & YH & TA2 \\
\hline Research concept and design & $\sqrt{ }$ & -- & -- & -- & -- & -- & -- & -- \\
\hline Collection and/or assembly of data & $\sqrt{ }$ & -- & $\sqrt{ }$ & $\sqrt{ }$ & $\sqrt{ }$ & $\sqrt{ }$ & -- & -- \\
\hline Data analysis and interpretation & $\sqrt{ }$ & $\sqrt{ }$ & -- & -- & -- & -- & -- & -- \\
\hline Writing the article & $\sqrt{ }$ & -- & -- & -- & -- & -- & $\sqrt{ }$ & -- \\
\hline Critical revision of the article & $\sqrt{ }$ & -- & -- & -- & -- & -- & $\sqrt{ }$ & $\sqrt{ }$ \\
\hline Final approval of article & $\sqrt{ }$ & $\sqrt{ }$ & $\sqrt{ }$ & $\sqrt{ }$ & $\sqrt{ }$ & $\sqrt{ }$ & $\sqrt{ }$ & $\sqrt{ }$ \\
\hline Statistical analysis & $\sqrt{ }$ & -- & $\sqrt{ }$ & -- & -- & -- & -- & -- \\
\hline Other (please specify)... & -- & -- & -- & -- & -- & -- & -- & -- \\
\hline
\end{tabular}

Publication history

Editor: Vikram C. Prabhu, Loyola University Medical Center, USA. EIC: Tadanori Tomita, Northwestern University Feinberg School of Medicine, USA.

Received: 20-Feb-2013 Revised: 26-Jun-2013

Re-Revised: 29-Aug-2013 Accepted: 05-Sep-2013

Published: 13-Sep-2013

\section{References}

1. Maesawa S, Fujii M, Nakahara N, Watanabe T, Saito K, Kajita Y, Nagatani T, Wakabayashi T and Yoshida J. Clinical indications for high-field 1.5 T intraoperative magnetic resonance imaging and neuro-navigation for neurosurgical procedures. Review of initial 100 cases. Neurol Med Chir (Tokyo). 2009; 49:340-9; discussion 349-50. I Article I PubMed

2. Arai T, Tanaka $T$ and Hasegawa $Y$ et al. A novel surgical procedure for brain tumors at The Jikei University School of Medicine Kashiwa Hospital: from the development of intraoperative magnetic resonance imaging to clinical usage. Tokyo Jikeikai Medical Journal. 2009; 124:169176.

3. Muragaki Y, Iseki H, Maruyama T, Kawamata T, Yamane F, Nakamura R, Kubo O, Takakura $\mathrm{K}$ and Hori T. Usefulness of intraoperative magnetic resonance imaging for glioma surgery. Acta Neurochir Suppl. 2006; 98:67-75. | Article | PubMed

4. Steinmeier R, Fahlbusch R, Ganslandt O, Nimsky C, Buchfelder M, Kaus M, Heigl T, Lenz G, Kuth R and Huk W. Intraoperative magnetic resonance imaging with the magnetom open scanner: concepts, neurosurgical indications, and procedures: a preliminary report. Neurosurgery. 1998; 43:739-47; discussion 747-8. | Article I PubMed

5. Wirtz CR, Knauth M, Staubert A, Bonsanto MM, Sartor K, Kunze S and Tronnier VM. Clinical evaluation and follow-up results for intraoperative magnetic resonance imaging in neurosurgery. Neurosurgery. 2000; 46:1112-20; discussion 1120-2. | Article | PubMed

6. Black PM, Moriarty T, Alexander E, 3rd, Stieg P, Woodard EJ, Gleason $\mathrm{PL}$, Martin CH, Kikinis R, Schwartz RB and Jolesz FA. Development and implementation of intraoperative magnetic resonance imaging and its neurosurgical applications. Neurosurgery. 1997; 41:831-42; discussion 842-5. I Article I PubMed

7. Kajiwara K, Yoshikawa K, Ideguchi M, Nomura S, Fujisawa H, Akimura T, Kato S, Fujii M and Suzuki M. Navigation-guided fence-post tube technique for resection of a brain tumor: technical note. Minim Invasive Neurosurg. 2010; 53:86-90. | Article | PubMed

8. Yoshikawa K, Kajiwara K, Morioka J, Fujii M, Tanaka N, Fujisawa H, Kato $S$, Nomura and Suzuki M. Improvement of functional outcome after radical surgery in glioblastoma patients: the efficacy of a navigationguided fence-post procedure and neurophysiological monitoring. $J$ Neurooncol. 2006; 78:91-7. | Article | PubMed 
Tanaka et al. Neuroscience Discovery 2013,

9. Karnofsky DA and Burchmal JH: The clinical evaluation of chemotherapeutic agents in cancer. In: MacLeod CM, ed. Evaluation of Chemotherapeutic Agents. 1949; 191-205.

10. Schneider JP, Trantakis C, Rubach M, Schulz T, Dietrich J, Winkler D, Renner C, Schober R, Geiger K, Brosteanu O, Zimmer C and Kahn T. Intraoperative MRI to guide the resection of primary supratentorial glioblastoma multiforme--a quantitative radiological analysis. Neuroradiology. 2005; 47:489-500. | Article | PubMed

11. Nimsky C, Ganslandt O, Hastreiter P, Wang R, Benner T, Sorensen AG and Fahlbusch R. Intraoperative diffusion-tensor MR imaging: shifting of white matter tracts during neurosurgical procedures--initial experience. Radiology. 2005; 234:218-25. | Article | PubMed

12. Ozawa N, Muragaki Y, Nakamura R, Hori T and Iseki H. Shift of the pyramidal tract during resection of the intraaxial brain tumors estimated by intraoperative diffusion-weighted imaging. Neurol Med Chir (Tokyo). 2009; 49:51-6. I Article I PubMed

13. Ohue S, Kumon Y, Nagato S, Kohno S, Harada H, Nakagawa K, Kikuchi K, Miki $\mathrm{H}$ and Ohnishi T. Evaluation of intraoperative brain shift using an ultrasound-linked navigation system for brain tumor surgery. Neurol Med Chir (Tokyo). 2010; 50:291-300. I Article I PubMed

14. Stummer W, Stocker S, Wagner S, Stepp H, Fritsch C, Goetz C, Goetz $A E$, Kiefmann R and Reulen HJ. Intraoperative detection of malignant gliomas by 5 -aminolevulinic acid-induced porphyrin fluorescence. Neurosurgery. 1998; 42:518-25; discussion 525-6. | Article I PubMed

15. Ahn JY, Jung JY, Kim J, Lee KS and Kim SH. How to overcome the limitations to determine the resection margin of pituitary tumours with low-field intra-operative MRI during trans-sphenoidal surgery: usefulness of Gadolinium-soaked cotton pledgets. Acta Neurochir (Wien). 2008; 150:763-71; discussion 771. | Article | PubMed

\section{Citation:}

Tanaka T, Ishii T, Kato N, Arai T, Akasaki Y, Yamamoto Y, Hasegawa $Y$ and Abe T. Wax pile method for glioma surgery utilizing intraoperative magnetic resonance imaging: a technical note. Neurosci Discov. 2013; 1:6. http://dx.doi.org/10.7243/2052-6946-1-6 